\title{
AUSÜBUNG DES INFORMATIONSRECHTS DER GMBH-GESELLSCHAFTER DURCH DRITTE IM DEUTSCHEN RECHT
}

Dr. Rauf KARASU

\section{A. Einleitung}

Nach $\S 51$ a Abs. 1 GmbHG haben die Geschäftsführer jedem Gesellschafter auf Verlangen unverzüglich „Auskunft über die Angelegenheiten der Gesellschaft zu geben" und die „Einsicht der Bücher und Schriften zu gestatten". Es handelt sich mithin um ein Individualrecht, welches der einzelne Gesellschafter unabhängig von der Gesellschaftergesamtheit, der Höhe seiner Kapitalbeteiligung und anderen Umständen ausüben kann. ${ }^{1}$

Während die Möglichkeit der Ausübung des Stimmrechts durch einen bevollmächtigten Dritten für Körperschaften in $\S \S 134$ Abs. 3 AktG. 47 Abs. $3 \mathrm{GmbHG}$ sowie $43 \mathrm{Abs}$. $5 \mathrm{GenG}$ vorgesehen wurde, sind entsprechende Regelungen für das Informationsrecht sowohl für Personengesellschaften als auch für Kapitalgesellschaften nicht vorhanden.

\section{B. Erfordernis der Ausübung des Informationsrechts durch Dritte}

Die Beteiligung eines Dritten an der Ausïbung des Informationsrechts kann in Form der Bevollmächtigung oder der Hinzuziehung eines Beistands (bei gleichzeitiger Anwesenheit des Gesellschafters selbst) erfolgen. Bevor die Zulässigkeit der Hinzuziehung eines Dritten zur Ausübung des Informationsrechts als Vertreter oder Beistand untersucht wird ${ }^{2}$, ist hier das Erfordernis der Ausiibung des Informationsrechts zu ermitteln.

\footnotetext{
'Kırıkkale Üniversitesi İktisadi ve İdari Bjlimler Fakültesi (LLM Heidelberg).

' Dazu eingehend Karasi. Informationsrecht des GmbH- Gesellschafters, S. 5 ff.

${ }^{2}$ Dazu eingehend unten $C$.
} 
Ein Gesellschafter kann beispielsweise aufgrund seines Alters, seines Gesundheitszustandes, seiner Abwesenheit am Sitz der Gesellschaft oder wegen fehlender Erfahrung in gesellschaftsrechtlichen und wirtschaftlichen Angelegenheiten daran gehindert sein, seine Mitgliedschaftsrechte sachgemäß wahrzunehmen. In diesen Fällen bedarf er der Ausübung seiner Mitgliedschaftsrechte und danit seines Informationsrechts durch Dritte.

Das Bedürfnis nach der Zulässigkeit des Dritten zur Ausübung des Informationsrechts soll durch folgende Beispielsfälle verdeutlicht werden:

\section{Fall 1:}

Ein Gesellschafter, der seinen Gesellschaftsanteil aufgrund einer Erbschaft übernommen hat, möchte die Gesellschaftspapiere, etwa die Bilanz und die Gewinn- und Verlustrechnung, auswerten. Da er aber in gesellschaftsrechtlichen und wirtschaftsrechtlichen Angelegenheiten unerfahren ist, kann er die Gesellschaftspapiere nicht richtig bewerten. Er ist nicht in der Lage, sich einen austeichenden Uberblick uiber die Gesellschaftsangelegenheiten zu verschaffen und sich eine eigene Meinung zu bilden. Er ist hinsichtlich der Ausuibung seines Informationsrechts in einer ähnlichen Lage wie der minderjährige Gesellschafter, dem aufgrund seines jugendlichen Alters die notwendige Ausbildung und Erfahrung fehlen. Daher ist er in diesem Fall, ebenso wie der Minderjährige, auf die Hinzuziehung eines Vertreters oder Beistands zur Ausübung seines Informationsrechts angewiesen.

\section{Fall 2:}

Ein Gesellschafter ist schwer krank bzw. körperlich gebrechlich oder muss sich wegen seiner vielfältigen wirtschaftlichen Aktivităten und Beteiligungen für eine längere Zeit vom Sitz der Gesellschaft entfernen. In diesen Fällen ist der Gesellschafter auf eine Vertretung angewiesen. Auch bei einer kurzen Abwesenheit kann er auf eine Vertretung angewiesen sein. Dies kann Z.B. der Fall sein, wenn während seiner kurzen Abwesenheit in der Gesellschafterversammlung über einen wichtigen Gegenstand z. B. über die Kapital- oder Strukturmaßnahmen, die für rechtliche und wirtschaftliche Interessen des Gesellschafters von erheblicher Bedeutung sind, entschieden wird. Da die Stimmrechtsvollmacht im GmbH-Recht nicht die Vollmacht zur Ausübung des Informationsrechts umfasst ${ }^{3}$, muss der Gesellschafter einc besondere Vollnacht für die Ausübung des Informationsrechts erteilen. Dies ergibt sich daraus, dass der zur Ausübung des Informationsrechts ausgewählte Dritte nach objektiven Kriterien Gewähr dafür bieten muss,

? Vgl. Tict7e. Informatjonsrechte, S. 22. 
dass das Gesellschaftsinteresse an der Geheimhaltung gesellschaftsinterner Angelegenheiten durch seine Kenntnis nicht geschädigt wird. Im Gegensatz zur Ausübung des Informationsrechts hat bei der bloßen Stimmabgabe die Person, die zur Stimmabgabe beauftragt wird, keine große Bedeutung, da bei der Ausuibung des Informationsrechts maßgebenden Gefahren bei der bloßen Stimmabgabe grundsätzlich nicht auftreten. ${ }^{4}$

Wird dem Gesellschafter bei den vorherigen Fällen die Möglichkeit der Ausübung seines Informationsrechts durch einen Vertreter oder Beistand verwehrt, so hat dies die gleiche Auswirkung wie der Ausschluss seines Informationsrechts. Die Schutzbedürftigkeit des betroffenen Gesellschafters überwiegt deshalb gegenüber den Interessen der Gesellschaft und der Mitgesellschafter an der Geheimhaltung der inneren Gesellschaftsangelegenheiten. ${ }^{5}$

\section{Zulässigkeit}

Nachdem das Erfordernis der Ausübung des Informationsrechts ermittelt wurde, ist nun zu untersuchen, ob die Hinzuziehung eines Dritten als Vertreter oder Beistand zulässig ist.

\section{Hinzuziehung eines Beistands}

Es ist allgemein anerkannt, dass ein Gesellschafter, soweit die Leitung der Einsicht in die Unterlagen der Gesellschaft bei ihm bleibt, berechtigt ist, einen geeigneten Sachverständigen bei der Ausübung seines Einsichtsrechts hinzuzuziehen, wenn er selbst nicht die nötige Sachkunde besitzt und die Hinzuziehung daher seinem gerechtfertigten Informationsinteresse dient ${ }^{6}$ Die Nichtzulassung des Beistands in diesem Fall würde zu einem Ausschluss des Informationsrechts führen, da derjenige Gesellschafter, der keine nötige Sachkunde besitzt, sein Informationsrecht ohne die Hilfe und Sachkenntnisse eines Sachverständigen nicht sachgerecht wahrnehmen könnte. ${ }^{\text {? }}$

Die überwiegende Ansicht vertritt die Auffassung ${ }^{8}$, dass die Gesellschafter einen Sachverständigen nicht nur bei Fehlen ihrer Sachkunde, sondern auch generell hinzuziehen dürfen. Dieser Ansicht ist zuzustimmen,

${ }^{4}$ Tietze, Tietze, Informationsrechte, S. 22.

${ }^{5}$ Vgl. Hirte, BB 1985, 2209; BGHZ 25, 115, 123 (zum Kommanditisten).

${ }^{6}$ Scholz/K. Schmidt, GmbHG \& 51 a Rn. 27; Hachenburg/Hiiffer, GmbHG, \& 51 a Rn. 16: Bopp, Informationsrechte, S. 52, 53; BayObLG, BB 1991, 1589; BGHZ 25, 115 (123); OLG Celle, ZIP 1983, 943; RGZ 25, 88; 148, 278; 170, 392; vgl. auch OLG Naumburg. GmbHR 1996, 936.

${ }^{7} \mathrm{Vgl}$. Hirte, BB 1985, 2209.

* Hachenburg/Hüffer, GmbHG, § 51 a Rn. 16: Scholz/K. Schmidt, § 51 a Rn. 27; Rowedder/Koppensteiner, GmbHG, § 51 a Rn. 3; Baumbach/Hueck/Zöllner, GmbHG, § 51 a Rn. 19. 
weil die Hinzuziehung eines Sachverständigen zum Zwecke der Beratung und Unterstützung eines Gesellschafters gegenüber der Stellvertretung eine geringere Belastung der übrigen Gesellschafter darstellt." Der Gesellschafter. nicht der Sachverständige. leitet in diesem Falle die Prüfung und er steht selbst der Gesellschaft gegenüber. ${ }^{10}$ Daher bleibt die Verantwortung ebenso wie auch das eigentliche Gesellschafterrecht voll und ganz bei dem Gesellschafter, so dass die gesellschaftsrechtliche Treuepflicht der Mitgesellschafter die Duldung des zugezogenen Sachverständigen gebietet."

Der Sachverständige kann von der Gesellschaft nur abgelehnt werden. wenn er nach objektiven Kriterien keine Gewähr für die Vertraulichkeic bietet $^{12}$. Dafür ist aber die Geselischaft darlegungs- und beweispflichtig..$^{13}$

\section{Ausibung durch Stellvertreter}

\section{Gesetziche Stellvertretung}

Wenn der Gesellschafter ein Minderjähriger, eine juristische Person oder eine Gesamthands- gesellschaft ist, kann er seine Rechte nicht selbst ausüben. In solchen Fällen muss sich der Gesellschafter zur Wahrung seiner Rechte zwangsläufig vertreten lassen. ${ }^{14}$ Daher ist es allgemein anerkannt. dass der gesetzliche Vertreter berechtigt ist, die Mitgliedschaftsrechte bzw. das Informationsrecht auszuüben." So sind die Insolvenzverwalter und Testamentsvollstrecker kraft Amtes befugt, das Informationsrecht auszuüben, sofern nicht in ihrer Person ein Verweigerungsgrund besteht. ${ }^{\text {to }}$

\section{Gewillkirte Stellvertretung}

Die Rechtsprechung ${ }^{17}$ hat bisher nur die Hinzuziehung des Sachverständigen (bei gleichzeitiger Anwesenheit des Gesellschafters selbst) zugelassen. Die Wahrnehmung des Informationsrechts allein durch einen

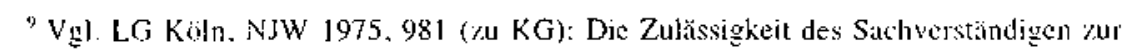
Ausübung des Informationsrechts kann als konkludent anerkannt werden. wenn die Stellvertretung bit dem Gesellschaltsvertrag zugelassen wird.

"'RG, DR 1944, 245, 246.

"VgI. BGHZ 25, 115 "r.

'Hachenhurg/Hüflier, GmbHG, \$ 51 a Rn. 17

"Hirte. BB 1985.2209 m.w.N.

"Bereits der Wortlaut ,gesetzliche Vertretung" besagt, dass der Betrollene seine Rechte nicht alleine ausuiben kann. und der Gesetzgeber deshalb eine Vertretung inordnete.

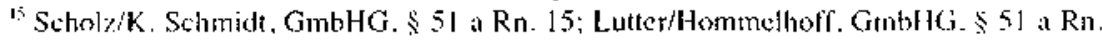
4: Baumbach/ Hucek/Zölner. GmbHG, \$ 51 a Rn. 5; Tietze. Informationsrectie, S. 22

"Schol\%/K. Schmidt, GmbHG. \$ \$I a Rn. 15; Lutter/Hommelhoff, GmbHG. \$ \$l a Ru. 4. 807.808

${ }^{17} \mathrm{Vgl}, \mathrm{BGHZ} 25.115,123: \mathrm{BGH}, \mathrm{BB}$ 1962, 899: BB 1979, 1315, 1316; WM 1984. 
Dritten (die Bevollmächtigung eines Dritten) wurde nur ausnahmsweise bei Vorliegen bestimmter Voraussetzungen ${ }^{18}$ für zulässig gehalten. Begründet wurde dies mit dem höchstpersönlichen Charakter des Informationsrechts, wonach die Gesellschafter ihre Informationsrechte persönlich ausüben müssen. Für die GmbH gilt aber diese Begründung nicht, denn das Informationsrecht des Gesellschafters aus § 51 a GmbHG ist im Gegensatz zu Personengesellschaften nicht höchstpersönlich. ${ }^{19}$ Das ergibt sich daraus, dass die $\mathrm{GmbH}$ nicht den Charakter einer grundsätzlich auf die Personen der Gesellschafter zugeschnittenen Gemeinschaft hat. ${ }^{20}$ Bei der GmbH sind zwar die persönlichen Bindungen stärker als bei der Aktiengesellschaft ${ }^{2 \prime}$, aber im Gegensatz zu den Personengesellschaften handelt es sich bei der $\mathrm{GmbH}$ um eine Gesellschaft mit eigener Rechtspersönlichkeit, die in ihrem Fortbestand von einem Gesellschafterwechsel unabhängig ist. Zu denken ist daran, dass die Stimmrechtsvollmacht nach $\$ 47 \mathrm{Abs}$. $3 \mathrm{GmbHG}$ und Fremdorganschaft nach $\S 6$ GmbHG bei der $\mathrm{GmbH}$ zulässig ist. Daher ist auch die Bevollmächtigung zur Ausübung des Informationsrechts wie die Hinzuziehung eines Sachverständigen grundsätzlich zulässig. ${ }^{22}$

\section{Person des Dritten (Personenkreis)}

\section{Allgemeines}

Da es über die Ausübung des Informationsrechts durch Dritte keine gesetzliche Vorschrift gibt, sondern deren Zulässigkeit durch Rechtsprechung und Literatur befürwortet wird, ist es fraglich, welche Personen als Dritte ausgewählt werden dürfen. In jedem Fall darf nicht jeder Dritte zur Ausübung des Informationsrechts zugelassen werden. Dies ergibt sich aus der Uberlegung, dass das Gesellschafts- interesse an der Geheimhaltung gesellschaftsinterner Angelegenheiten durch Kenntnis des Dritten geschädigt wird.

Liegt in der Person des Dritten einen Grund vor, der einer Offenbarung der Gesellschaftsinterna ${ }^{23}$ entgegensteht, kann die Ausübung des

${ }^{18}$ Z.B. bei der Krankheit des einsichtsbegehrenden Gesellschafters (BGHZ 25, 115 , 123), bei der Zustimmung der übrigen Gesellschafter (BGH. WM 1961 1329).

${ }^{19}$ Lutter/Hommelhoff, GmbHG, § 51 a Rn. 4; Baumbauch/Hucck/Zöllner. GmbHG. \& 51 a Rn. 5.

${ }^{2 n}$ Hachenburg/Hüffer, GmbHG, \$ 51 a Rn. 16.

${ }^{2}$ Z.B. ist die Übertragbarkeit der Anteile erschwert.

22 Hachenburg/Hüffer, GmbHG. § 51 a Rn. 16; Baumbach/Hueck/Zöllner, GmbHG. \& 51 a Rn. 5; Bitter, ZIP 1981, 825, 828.

${ }^{27} \mathrm{Zu}$ den Intema der Gesellschaft eingehend siehe Karasu. Informationsrecht des GmbH-Gesellschafters, S. $90 \mathrm{ff}$. 
Informationsrechts durch diesen Dritten die Gefahr einer Schädigung der Gesellschaft mit sich bringen, weil das Informationsrecht weitreichende Einblicke in die Interna der Gesellschaft eröffnet. So kann z.B. der zur Gesellschaft in einem Wettbewerbsverhältnis stehende Vertreter Kenntnis von Produktionsgeheimnissen, von Kundenstamm und von Geschäftsvorhaben erfahren. Bei der personalistisch geprägten $\mathrm{GmbH}$ ist die vertrauensvolle Zusammenarbeit der Gesellschafter besonders wichtig. Folglich ist das Interesse der Gesellschafter an der Abwehr fremder Einflüsse groß.

Die Geheimhaltungsinteressen der Gesellschaft können dadurch gewährleistet werden, dass der zur Ausübung des Informationsrechts ausgewählte Dritte nach objektiven Kriterien Gewähr dafür bieten kann, dass das Gesellschaftsinteresse an der Geheimhaltung gesellschaftsinterner Angelegenheiten durch seine Kenntnis nicht geschädigt wird. Es darf sich nicht um ungeeignete Personen, wie z.B. Konkurrenten, handeln, durch die eine Schädigung der Gesellschaftsinteressen eintreten kann. Als geeignet sind die Mitgesellschafter und die Personen, die zur Berufsverschwiegenheit verpflichtet sind, anzusehen, weil die Gefahr, dass die Informationen aus der Gesellschaft hinaus getragen werden, sicherlich am besten nur dann verhindert werden kann, wenn den Personenkreis, der Zugang zu den Informationen hat, möglich klein hält. ${ }^{24}$ Aus diesem Grund ist der Ansicht ${ }^{25}$ nicht zu folgen, wonach die Gesellschaft den Bevollmächtigten Dritter nur dann ablehnen darf, wenn sie beweist, dass das Auftreten des Dritten in der Gesellschaft aus in seiner Person liegenden Gründen nicht geduldet werden kann. Diese Ansicht sieht daher auch solche Personen als zulässig, bei denen wegen ihrer Vertrauenswürdigkeit oder Interessenlage angenommen werden muss, dass sie zur Verschwiegenheitspflicht in gleicher Weise gehalten sind wie der Gesellschafter. ${ }^{26}$ So können z.B. Prokuristen und Nießbratucher von der Gesellschaft nicht abgelehnt werden. ${ }^{27}$

Im Gegensatz zum Einsichtsrecht hat bei der Auskunftserteilung die Person, die die Auskunft verlangt, keine große Bedeutung, da bei der Erteilung der Auskunft der inhaltliche Umfang der Antwort von der Gesellschaft bestimmt wird. ${ }^{2 *}$

\footnotetext{
IA Tietze. Informationsrechte. S. 20 f.

${ }^{25} \mathrm{Vgl}$. Hirte. BB 1985, 2209; Hachenburg/Hüfler, GmbHG, \$ 51 a Rn. 17: Scholz/K. Schmidt, GmbHG、 5 I a Rn. 15.

"Hachenburg/Hüffer, GmbHG. \$ 51 a Rn. 17: Scholz/K. Schmidt, GinbHG, \$ 51 a Rn. 15.

${ }^{27}$ Hachenhurg/Hüffer. GmbHG. § 51 a Rn. 17; Scholz/K. Schmidı, GmbltG. \$̦ 51 a Rn. 15.

${ }^{2 x} \mathrm{Vgl}$. auch Hirte, BB 1985, 2208.
} 


\section{Mitgesellschafter}

Ein Mitgesellschafter als Vertreter oder Beistand ist zulässig, sofern nicht auch ihm gegenüber ein Verweigerungsgrund besteht. ${ }^{29}$

\section{Berufsmäßig geeignete Dritte}

Außer den Mitgesellschaftern können auch die Personen, die zur beruflichen Verschwiegenheit verpflichtet sind, ausgewählt werden ${ }^{30}$ Unter diese Personen fallen Wirtschaftsprüfer. Steverberater, Rechtsanwälte, Notare und vereidigte Buchprüfer. Durch die Auswahl dieser vertrauenswürdigen und sachkundigen Personen wird das Interesse der Gesellschaft bzw. der übrigen Gesellschafter gewahrt ${ }^{31}$, weil diese kraft ihrer Stellung, Bestellung oder Vereidigung zur beruflichen Verschwiegenheit verpflichtet sind.

Bei der Einsichtnahme ist die Gesellschaft verpflichtet, auch die Mitarbeiter des sachverständigen Dritten zuzulassen, weil bei der Einsicht in die Unterlagen im Regelfall eine große Menge von Daten aufzunehmen ist. ${ }^{32}$ Dabei handelt es sich um eine routinemäßige Tätigkeit, für deren Ausübung es nicht der beruflichen Qualifikation eines Sachverständigen bedarf, die vielmehr auch von dessen Mitarbeitern unter seiner Leitung und Aufsicht durchgeführt werden kann.$^{33}$ Da die Gehilfen der Sachverständigen auch zur Verschwiegenheit verpflichtet $\operatorname{sind}^{34}$ und die Verletzung dieser Verschwiegenheitspflicht nach $\$ 203$ Abs. 3 S. 1 i.V.m. Abs.1 Nr. 3 StGB bestraft wird, wird dem Interesse der Gesellschaft an einer Geheimhaltung ihrer Unterlagen in ausreichender Weise Rechnung getragen. ${ }^{35}$

\section{Einwendungen gegen die Person des Dritten}

Einwände gegen die Person des Dritten werden nur berücksichtigt, wenn die Gesellschaft diese darlegt und gegebenenfalls beweist, dass ihr Geheimhaltungsinteresse durch den Dritten nicht gewahrt wird. Wenn z.B.

${ }^{24}$ Scholz/K. Schmidt, GmbHG, \$ 51 a Rn. 15; Tietze, Informationsrechte, S. 20 f.: v. Bitter, BB 1981, 825, 828.

${ }^{30}$ Scholz/K. Schmidt. GmbHG, § 51 a Rn. 27; Baumbach/Hueck/Zöllner. GmbHG, § 51 a Rn. 5; Lutter/Hommethoff, GmbHG. \$ 51 a Rn. 4; Goerdeler. FS für Stimpel, S. 125 f.; Tietze, Informationsrechte, S. 19; BGH, BB 1984, 1274.

${ }^{31}$ Für die Frage, ob ein Wirtschaftspriufer oder Steuerberater geeignet wäre, vgl. Goerdeler, FS für Stimpel, S. 125, 135.

${ }^{32}$ OLG Düsseldorf, BB 1995, $143 \mathrm{f}$.

${ }^{33}$ OLG Düsseldorf. BB 1995, 143 f.

${ }^{34} \mathrm{Vgl}$ \& 50 WPO.

${ }^{35}$ OLG Düsseldorf, BB 1995, 143 f. 
die zur Ausübung des Informationsrechts ausgewählte Person einem Konkurrenzunternehmen angehört oder einzelne Gesellschafter beleidigl hat ${ }^{3 / 2}$, kann dieser Vertreter oder Sachverständige abgelehnt werden.

Die Ablehnung eines ausgewählten Dritten kann jedoch nicht darauf gestutzt werden, dass dieser mit dem informationsberechtigten Gesellschafter eng verbunden ist. So ist $2 . B$. der Fall denkbar, dass ein Gesellschafter bei der Ausübung seines Informationsrechts einen Rechtsanwalt hinzuzieht, der ihn zur gleichen Zeit als Prozessbevollmächtigter bei der Wahtnehmung seiner Interessen gegenüber der Gesellschaft unterstützt. In diesem Fall darf der Rechtsanwalt nicht abgelehnt werden, denn auch der Gesellschafter selbst muss grundsätzlich nicht sein Informationsrecht hinter die Interessen der Gesellschaft zurückstellen, weil das Informationsrecht des Gesellschafters aus $\$ 51$ a GmbHG ein eigennütziges Recht ist. ${ }^{37}$ Anderes gilt, wenn der Dritte durch sein Auftreten zum Unfrieden in der Gesellschaft geführt hat.

\section{E. Satzungsbestimmung über die Ausübung des Informationsrechts durch Dritte}

Das Gesellschaftsrecht enthält, wie oben dargelegt, zur Frage der Ausubung des Informationsrechts durch einen Dritten keine ausdrickliche gesetzliche Regelung. Dies führt dazu, dass die Ausubung des Informationsrechts durch Dritte weitgehend den gesellschaftsvertraglichen Bestimmungen iberlassen ist. Wegen dieser Rechtsunsicherheit streiten die Gesellschafter öfter über die Zulässigkeit oder Unzulässigkeit des Informationsrechts durch einen Dritten und dies beeinträchtigt natürlich den Gesellschaftsfrieden. Deshalb ist eine Untersuchung der Zulässigkeit der Ausübung durch Dritte im Gesellschaftsvertrag erforderlich. Zu klären ist demnach insbesondere die Frage, ob die Satzung die Beteiligung eines Dritten zur Ausübung des Informationsrechts zwingend vorschreiben oder verbieten kann.

\section{Zwingende Dritbeteiligung durch Satzung}

Das Informationsrecht aus $\$ 51$ a GmbHG steht dem Gesellschalter persönlich 7u, auch wenn er dazu berechtigt ist, die Ausübung seines Informationsrechts durch Dritte vertreten zu lassen. Die Satzung kann daher die Gesellschafter grundsätzlich nicht verpflichten, auf ihr Recht zur persönlichen Ausübung zu verzichten. Solch eine Vereinbarung greift in

\footnotetext{
Wh Wiedemann, lihertragung. S. 352: BGH. WM 1962,883.

${ }^{37} \mathrm{Vgl}$. Kalasu, Informationsrecht des GmbH-Gesellschafters. S. 19911.
} 
den unentziehbaren Kern des Informationsrechts ein. ${ }^{38}$ Ein Gesellschafter darf daher trotz Bestehens einer Satzungsklauseln, die einen Gesellschafter von der persönlichen Ausübung seines Informationsrechts ausschließ3t, sein Informationsrecht selbst ausüben. ${ }^{39}$

Eine besondere Problematik kann sich allerdings dann ergeben, wenn der informationsbegehrende Gesellschafter gleichzeitig auch Wettbewerber der Gesellschaft is ${ }^{40}$. Dabej besteht tatsächlich die Gefahr, dass die zur Gesellschaft im Wettbewerb stehende Gesellschafter sein Informationsrecht zugunsten des Konkurrenzunternehmens und damit zum Nachteil der zur Information verpflichteten Gesellschaft ausnutzt. ${ }^{4 \mid}$ In diesem Fall ist die Ausübung des Informationsrechts durch den Gesellschafter selbst für die Gesellschaft unzumutbar und es besteht daher ein Bedürfnis, das Informationsrecht des Konkurrentgesellschafters zu beschränken. Aber er muss sich wie seine Mitgesellschafter iber die Lage seiner Gesellschaft informieren, um seine Mitgliedschaftsrechte sachgemäß auszuüben. Sonst wird er vom Gesellschaftsleben ausgeschlossen. So bietet sich als Ausweg die Vertretung des Gesellschafters bei der Ausübung des Informationsrechts. In der Satzung kann daher vorgeschrieben werden, dass die mit der Gesellschaft im Wettbewerb stehenden Gesellschafter ihre Informationsrechte durch einen zur Verschwiegenheit verpflichteten Treuhänder, z.B. durch einen Wirtschaftsprüfer, ausüben. Der Treuhänder soll in diesem Fall dem Gesellschafter, für den er auftritt, die Information über die Vorgänge oder Sachverhalte ohne Erwähnung von Einzelheiten weitergeben. ${ }^{22}$ Dieser Sachverständige soll aber sowohl für den Gesellschafter als auch für die Gesellschaft gleichermaßen vertrauenswürdig sein, damit die Interessen beider Seiten gewahrt werden können. Deshalb soll das Recht auf Auswahl des Treuhänders nicht dem Konkurrenzgesellschafter zustehen, sondern die Gesellschaft und der Gesellschafter sollen sich auf einen Treuhänder einigen. Ansonsten bestünde die Besorgnis, dass der Treuhänder die gesperrten Informationen an den

\footnotetext{
${ }^{38} \mathrm{Vgl} . \$ 51$ a Abs. $3 \mathrm{GmbHG}$.

${ }^{3}$ Zur gesetzlichen Regelung für die Zwangsvertretung im Genossenschaftsrecht vgl. \$ 43 a Abs. 1 GenG.

* Anders als für den persönlich haftenden Gesellschafter, existiert im Recht der GmbH für den Gesellschaíter kein gesetzlich normiertes Wettbewcrbsverbot. Trotz. des Fehlens ciner ausdrücklichen gesetzlichen Regelung unterliegt ein GmbH-Gesellschafter nach überwiegender Ansicht einem Wettbewerbsverbot kraft Treuepflicht. Unstritten ist jedoch die Frage, unter welchen Voraussetzungen und in welchem Umfang dies der Fall ist. Umstritten ist vor allem die Frage, ob alle Geselischafter, auch die Minderheitsgeselischafter, oder nur die herrschenden Gesellschafter einem ungeschriebenen Wettbewerbsverbot unterliegen. Dazu eingehend Karasu. Informationsrecht des GmbH- Gesellschafters, S. 151 ff.

"Dazu cingehend Karasu. Informationsrecht des GmbH. Gesellschafters, S. 95 ff.

s2 Goerdeler, FS für Stimmpel, S. 125. 129; Tietze. Informationsrechte, S. 77.
} 
Konkurrenzgesellschafter weiterleitet. ${ }^{43}$ Durch Satzung kann das Auswahlrecht einem Dritten ${ }^{44}$ oder der Handelskammer ${ }^{4.5}$ übertragen werden. Fehlt eine solche Bestimmung in der Satzung oder konnten die Parteien sich auf einen Sachverständigen nicht einigen, so kann nach $\$ 145$ FGG das Amtsgericht angerufen werden. ${ }^{46}$

Der Konkurrenzgesellschafter soll auch nicht den Treuhänder frei abberufen dürfen. Sonst wäre zu besorgen, dass sich der Treuhänder unzulässigen Informationswünschen des Konkurrenzgesell- schafters nicht genügend zu widersetzen vermag. ${ }^{47}$

\section{Ausschluss der Drittbeteiligung durch Satzung}

Fraglich ist, ob in der Satzung die Möglichkeit der Ausübung des Informationsrechts durch einen Dritten ganz ausgeschlossen werden darf. Besonders bei den Familiengesellschaften findet man häufig eine Bestimmung, dass jeder Gesellschafter seine Mitgliedschaftsrechte persönlich ausüben muss, um die Gesellschaft vor dem Eindringen des Fremden zu schützen, weil bei diesen Gesellschaften das Bedürfnis nach Fernhaltung von Dritten von inneren Gesellschaftsangelegenheiten stärker als bei den anderen Gesellschaften ist.

Die Gesellschafter, die durch Satzung von der Ausübung des Informationsrechts durch Dritte ausgeschlossen wurden. nehmen damit zwar bewusst eine Beschränkung ihres Informationsrechts hin. Aber trotz dieser vertraglichen Vereinbarung können die Gesellschafter der $\mathrm{GmbH}$ ihre Informationsrechte durch Vertreter oder Beistand ausüben lassen, wenn sie ihr Informationsrecht aus unabwendbaren Gründen (geschäftliche Unerfahrenheit, Krankheit bzw. Gebrechlichkeit und Abwesenheit) nicht selbst oder nicht sachgemäß ausüben können, weil beim Vorhandensein dieser Gründe eine solche Vereinbarung für die Gesellschafter die gleiche Auswirkung hat wie ein Ausschluss des Informationsrechts selbst und daher in den Kernbereich des Informationsrechts eingreift. Da der Kernbereich des Informationsrechts unverzichtbar ist und daher auch durch Satzung davon nicht abgewichen werden $\mathrm{kann}^{48}$, ist der Ausschluss der Dritten nur insoweit

${ }^{43}$ Ivens, GmbHR 1989.276; Baumbach/Hueck/Zöllner, GmbHG, \$ 51 a Rn. 25a.

+ Vgl. Ivetis. GimbHR 1989.273, 276; Gustavus. GmbHR 1989. 181. 184.

${ }^{45} \mathrm{Vgl}$. BGiHZ $44,158$.

4h Ivens, GmbHR 1989, 277; Baumbach/Hueck/Zöllner, GmbHG, s\$ 51 a Rn. 25a: BGH. BB 1970, 187; OLG Frankfurt, GmbHR 1995, 904.

${ }^{47}$ [vens, GmbHR 1989. 276.

${ }^{4 *} \mathrm{Vgl}$. 5 I a Abs. $3 \mathrm{GmbHG}$. 
zulässig, als der Ausschluss des Informationsrechts überhaupt gestattet ist. ${ }^{49}$

Für die Beurteilung der Ausnahmefälle, in denen die Ausübung des Informationstechts durch einen Dritten auch bei Vertreterklauseln für zulässig gehalten wird, bestehen keine klaren Abgrenzungskriterien. Fraglich ist z.B., wie man den Grad der Dringlichkeit einer Reise oder die Schwere einer Erkrankung bestimmen kann. Die Bewertung dieser Fälle ist wegen der mangelnden Nachprüfbarkeit als problematisch einzuschätzen.

Im Gesellschaftsvertrag können aber Anforderungen an die Person des Dritten gestellt und damit der Kreis der zur Ausübung des Informationsrechts zugelassenen Personen eingeengt werden. Zulässig sind dabei Regelungen der Art, dass nur die Personen zugelassen werden, die beruflich zur Verschwiegenheit verpflichtet sind. ${ }^{50}$

Der Umfang der Ausübungsbefugnis des Bevollmächtigten wird durch den Inhalt des Informa- tionsrechts bestimmt. Der Bevollmächtigte ist im Rahmen seiner Vertretungsmacht befugt, sich wie der von ihm vertretene Gesellschafter zu verhalten. Es empfehlt sich, Gegenstand und Umfang der Voll- macht genau zu umschreiben, damit sie ohne Rückfragen nachgeprüft und den anderen Gesellschaftern nachgewiesen werden können. Daher soll die Vollmacht, wie es in $\S 47$ Abs. 3 GmbHG und $\S 134$ Abs. $3 \mathrm{~S} .2 \mathrm{AktG}$ zur Stimmrechtsvollmacht vorgesehen ist, in schriftlicher Form erteilt werden. ${ }^{51}$

\section{F. Zusammenfassung}

Das Informationsrecht aus $\S 51$ a GmbHG ist ein von dem Stimmrecht und von der Höhe der Beteiligung unabhängiges Mitgliedschaftsrecht und steht grundsätzlich nur dem Gesellschafter zu. Höchstpersönliche Ausübung ist allerdings nicht geboten. Der Gesellschafter kann sein Informationsrecht durch einen Vertreter oder einen Beistand ausüben. Der zur Ausübung des Informationsrechts auszuwählende Dritte muss aber die Gewähr dafür bieten, dass das Gesellschaftsinteresse an der Geheimhaltung der Gesellschaftsinterna durch seine Kenntnis nicht gefährdet wird. Zulässig sind daher nur die Mitgesellschafter und zur Berufsverschwiegenheit verpflichtete Sachverständige.

$\mathrm{Da}$ das Informationsrecht aus $\S 51$ a $\mathrm{GmbH}$ dem Gesellschafter

19 Saenger, Beteiligung Dritte, S, 157; Scholz/K. Schmidt, GmbHG, \&47 Rn. 96. Rn. 19.

K. Schmidt, Informationsrechte. S. 25; Baumbach/Hueck/Zöllner, GmbHG, \$ 51 a

"Saenger, Beteiligung Dritte, S. 193. 
persönlich zusteht, auch wenn er die Ausübung seines Rechts durch Dritte vertreten lassen darf, kann die Satzung den Gesellschafter grundsätzlich nicht verpflichten, auf sein Recht zır persönlichen Ausübung zu verzichten. Zulässig ist aber eine Satzungsklausel, die einen Konkurrentgesellschafter von der persönlichen Ausubbung seines Informationsrechts ausschlielit und statt dessen die Einschaltung eines zur Verschwiegenhejt verpflichteten Dritten vorsieht. Damit wird die Ausnutzung des Informationsrechts durch die Gesellschafter, die zur Gesellschaft im Wettbewerb stehen, verhindert. Zulässig ist auch eine Satzungsbestimmung, die die Möglichkeit der Ausübung des Informationsrechts durch einen Dritten ganz ausschließt. Die Gesellschafter können aber trotz solcher Satzungsbestimmungen ihre Informationsrechle durch Dritte ausüben lassen, wenn sie ihr Informationsrecht aus unabwendbaren Gründen nicht selbst oder sachgemäß ausüben können. 


\section{LITERATURVERZEICHNIS}

Baumbach, Adolf/Hueck, Alfred; GmbH-Gesetz, 17. erweiterte und völlig überarbeitete Auflage, München 2000 und 13. Auflage, München 1970 (zit.: „Baumbach/ Hueck/Berarbeiter, GmbHG,...“ bzw. „Baumbach / Hueck / Berarbeiter, GmbHG, 13. Aufl.,...")

Bitter, Christian von; Das Informationsrecht der GmbH-Gesellschafter in $\S \S 51$ a, 51 b GmbHG, ZIP 1981, S. 825-832

Bopp, Hans-Peter; Die Informationsrechte des GmbH-Gesellschafters Eine rechtsvergleichende Untersuchung des deutschen, französischen und schweizerischen Rechts -. Konstanz 1991 (zit.: „Bopp, Informationsrechte,...")

Goerdeler, Reinhard; Die Zuziehung von Sachverständigen bei der Einsicht in die Bücher, in: Festschrift für Walter Stimpel, Berlin-New York 1985, S. 125-137 (zit.: „Goerdeler, FS für Stimpel,...")

Hachenburg, Max; Gesetz betreffend die Gesellschaft mit beschränkte Haftung - Großkommentar, 1. Band ( $\S$ 1-34) 8. neubearbeitete Auflage 1992; 2. Band ( $\S \S 35-52), 8$. neubearbeitete Auflage 1997; 2. Band ( $\$ 35$ 52), 7. Auflage 1970; 3. Band ( $\$ \$ 53-85)$, 8. neubearbeitete Auflage, BerlinNew York 1997 (zit.: „Hachenburg/ Bearbeiter, GmbHG...." bzw. „Hachenburg/Bearbeiter, GmbHG, 7. Aufl., ...")

Hirte, Heribert; Die Ausubung der Informationsrechte von Gesellschaftern durch Sachverständige, BB 1985, S. 2208-2210

Ivens, Michael; Das satzungsmäßige Konkurrenzverbot für $\mathrm{GmbH}$ Gesellschafter - Der Konflikt der Konkurrenzbeziehung und dessen Lösung durch einen satzungsmäßig geregelten Anspruch auf Unterlassung der Konkurrenzbeziehung unter Berücksichtigung der kartellrechtlichen Problematik -, Hamburg 1987 (zit.: „Ivens, Das satzungsmäßige Konkurrenzverbot,...")

Karasu, Rauf; Informationsrecht des GmbH-Gesellschafters unter besonderer Berücksichtigung der Lösungsmöglichkeiten zur Unangemessenheit des $\S 51$ a GmbHG, Diss. Konstanz, 2003 (zit.: Karasu. „Informationsrecht des GmbH-Gesellschafters...")

Lutter, Marcus/Hommelhoff, Peter; GmbH-Gesetz Kommentar, 15. neubearbeitete und erweiterte Auflage, Köln 2000 (zit.: „Lutter / Hommelhoff, GmbHG....")

Rowedder, Heinz; Gesetz betreffend die Gesellschaften mit beschränkter Haftung, Kommentar, 3. neubearbeitete Auflage, München 1997 (zit.: „Rowedder/Bearbeiter, GmbHG ,...") 
Saenger, Ingo; Beteiligung Dritter bei Beschlußfassung und Kontrolle im Gesellschaftsrechts, Berlin 1990 (zit.: „Saenger, Beteiligung Dritter,...")

Scholz, Franz; Kommentar zum GmbH-Gesetz, Band 1 (\$\$ 1-44), 9. neubearbeitete und erweiterte Auflage, Köln 2000 und 7. neubearbeitete Auflage, Köln 1986: Band 2 ( $\$ \$ 45-85$ ), 8. neubearbeitete und wesentlich erweiterte Auflage, Köln 1995 (zit.: „Scholz/Bearbeiter, GmbHG ...." bzw. Scholz/Bearbeiter, GmbHG, 7. Aufl.,...")

Tietze, Jörg; Die Informationsrechte des GmbH-Geselischafters, Köln 1985 (zit.: „Tietze, .,Informationsrechte...")

Wiedemann, Herbert; Die Übertragung und Vererbung von Mitgliedschaftsrechten bei Handelsgesellschaften, München-Berlin 1965 (zit.. ,Wiedemann, Übertragung,...") 


\section{\$1. ABKÜRZUNGSVERZEICHNIS}

a.A.

Abs.

AcP

a.F.

$\mathrm{AG}$

AktG

Anh.

Anm.

Art.

Aufl.

BayObLG

$\mathrm{BB}$

$\mathrm{Bd}$.

Begr.

BGB

BGBl.

BGH

BGHZ

bspw.

BT-Drucks.

BVerfG

bzgl.

bzw.

DB

ders.

d.h.

Diss.

f.

ff.

FN

FS

GbR

gem.

GenG

GG

$\mathrm{GmbH}$

GmbHG

GmbHR

GWB

h.A. anderer Ansicht

Absatz

Archiv für die civilistische Praxis alte Fassung

Aktiengesellschaft, Die Aktiengesellschaft

Aktiengeset $z$

Anhang

Anmerkung

Artikel

Auflage

Bayerisches Oberstes Landesgericht

Der Betriebsberater

Band

Begründung

Bürgerliches Gesetzbuch

Bundesgesetzblatt

Bundesgerichtshof

Entscheidungen des Bundesgerichtshofs in

Zivilsachen

beispielsweise

Bundestagsdrucksache

Bundesverfassungsgericht

bezüglich

beziehungsweise

Der Betrieb

derselbe

das beißt

Dissertation

folgende

fortfolgende

Fußnote

Festschrift

Gesellschaft bürgerlichen Rechts

gemäß

Gesetz betreffend die Erwerbs- und Wirtschaftsgenossenschaften

Grundgesetz

Gesellschaft mit beschränkter Haftung

Gesetz betreffend die Gesellschaften mit beschränkter Haftung

GmbH-Rundschau

Gesetz gegen Wettbewerbsbeschränkungen

herrschende Ansicht 


\begin{tabular}{|c|c|}
\hline HGB & Handelsgesetzbuch \\
\hline h.M. & herrschende Meinung \\
\hline Hrsg. & Herausgeber \\
\hline i.e.S. & im engeren Sinne/engeren Sinn \\
\hline $\mathrm{i} . d . \mathrm{R}$. & in der Regel \\
\hline i.S. & im Sinne \\
\hline i.V.m. & in Verbindung mit \\
\hline JuS & Juristische Schulung \\
\hline $\mathrm{JZ}$ & Juristenzeitung \\
\hline $\mathrm{KG}$ & Kammergericht; Kommanditgeselischaft \\
\hline $\mathrm{KGaA}$ & Kommanditgesellschaft auf Aktien \\
\hline Komm. & Kommentar \\
\hline LG & Landgericht \\
\hline n.F. & neue Fassung \\
\hline NJW & Neue Juristische Wochenschrift \\
\hline NJW-RR & NJW-Reclitsprechungs-Report \\
\hline $\mathrm{Nr}$ & Nummer \\
\hline NZG & Neue Zeitschrift für Gesellschaft \\
\hline $\mathrm{OHG}$ & Offene Handelsgesellschaft \\
\hline OLG & Oberlandesgericht \\
\hline RefE. & Referentejentwurf \\
\hline RegE. & Regierungsentwurf \\
\hline RG & Reichsgericht \\
\hline RGZ & Reichsgericht, Entscheidungen in Zivilsachen \\
\hline Rn. & Randnummer \\
\hline S. & Seite \\
\hline StGB & Strafgesetzbuch \\
\hline u.a. & unter anderem \\
\hline UmwG & Umwandlungsgesetz \\
\hline u.s.w. & und so weiter \\
\hline UWG & Gesetz gegen den unlauteren Wettbewerb \\
\hline $\mathrm{v}$ & von; vom \\
\hline vgl. & vergleiche \\
\hline Vorb. & Vorbemerkung \\
\hline WM & Wertpapier-Mittejlungen \\
\hline WPg & Die Wirtschaftsprüfung \\
\hline WuW & Wirtschaft und Wettbewerb \\
\hline z.B. & zum Beispiel \\
\hline ZGR & $\begin{array}{l}\text { Zeitschrift für Unternehmens- and } \\
\text { Gesellschaftsrecht }\end{array}$ \\
\hline ZHR & $\begin{array}{l}\text { Zeitschrift für das gesante Handelsrecht und } \\
\text { Wirtscliaftsrecht }\end{array}$ \\
\hline ZIP & Zeitschrift für Wirtschaftrecht und \\
\hline zit. & zitiert \\
\hline
\end{tabular}

